\title{
Associations of LBX1 gene and adolescent idiopathic scoliosis susceptibility: a meta- analysis based on 34,626 subjects
}

\author{
Yaqin Cao, Jikang Min * ${ }^{*}$, Qianghua Zhang, Heng Li and Haidong Li
}

\begin{abstract}
Background: The results of studies investigating the association between the ladybird homeobox 1 (LBX1) gene polymorphisms and the risk of adolescent idiopathic scoliosis (AIS) are not all the same. As such, we performed a meta-analysis to estimate the association between $\angle B X 1$ gene polymorphisms and AIS susceptibility.

Methods: Relevant studies published before 15 November 2015 were identified by searching PubMed, EMBASE, ISI web of knowledge, EBSCO, CNKI and CBM. The strength of relationship was assessed by using odds ratios (ORs) and $95 \%$ confidence interval (Cl).

Results: A total number of eight case-control studies including 10,088 cases and 24,538 controls were identified. The results showed that T allele of rs 111090870 increased AIS susceptibility in Asians (T vs. C, OR=1.22, $95 \%$ Cl: 1. 16-1.29, $P<0.001$ ), Caucasians ( $T$ vs. $C, O R=1.17,95 \%$ Cl: 1.14-1.21, $P<0.001$ ) and in female (T vs. C, OR=1.21, $95 \%$ $\mathrm{Cl}: 1.17-1.25, P<0.001)$. The $\mathrm{G}$ allele of $\mathrm{rs} 678741$ decreased AIS risk in female ( $\mathrm{G}$ vs. $\mathrm{A}, \mathrm{OR}=0.83,95 \% \mathrm{Cl}: 0.81-0.85$, $P<0.001$ ), and the $G$ allele of the rs625039 increased AlS susceptibility in Asians (G vs. $A, O R=1.14,95 \%$ Cl: 1.11-1. $17, P<0.001)$.
\end{abstract}

Conclusions: Our meta-analysis provides evidence that rs111090870, rs678741 and rs625039 polymorphisms near LBX1 gene are associated with AIS susceptibility in some populations. However, our findings are based on only a limited number of studies.

Keywords: LBX1 gene, Adolescent idiopathic scoliosis, Gene polymorphism, Meta-analysis

\section{Background}

Adolescent idiopathic scoliosis (AIS) is a medical condition which affects $1-4 \%$ of children in the at-risk population of those aged 10-16 years, using a cut-off point of $10^{\circ} \mathrm{Cobb}$ angle or more [1,2]. An X-ray of an AIS patient is showing in Additional file 1: Figure S1. Without a known cause, AIS has been simply defined as a structural lateral curvature. However, the deformity is three-dimensional, which entails the coronal, sagittal and transverse planes of the spine $[3,4]$. AIS leads to significant functional disabilities, especially pulmonary impairment $[5,6]$. It also causes pain and cosmetic problems $[7,8]$. The cause of AIS is complex and possible ethiology pathogeneses include genetic factors, hormones and metabolic dysfunction,

\footnotetext{
* Correspondence: jikangminjkm@sina.cn

Department of Orthopaedics, the First People's Hospital of Huzhou, 158 Guangchanghou Road, Huzhou, Zhejiang Province 313000, China
}

abnormal growth, and environmental and life style factors [9-12]. Of all these factors, genetic factors are widely-accepted and well-documented [13-15]. Many genes involving in the initiation and evolution of AIS have been identified as its susceptible genes, such as Melatonin Receptor 1B (MTNR1B), ladybird homeobox 1 (LBX1), tryptophan hydroxylase 1 (TPH1), arylalkylamine $\mathrm{N}$-acetyltransferase $(A A-N A T)$ and Basonuclin 2 (BNC2) [1, 16-18], and among them, the $L B X 1$ gene is widely investigated.

The $L B X 1$ gene locates on chromosome 10q24.31, and it is a hemeobox transcription factor $[19,20]$. It takes part in spinal cord differentiation and patterning, and somatosensory signal transduction. Therefore, $L B X 1$ is a strong biological candidate gene for AIS [21, 22]. LBX1 encodes ladybird homeobox 1, orthologous to Drosophila ladybird-late, which plays a key role in regulation of 
muscle precursor cell migration and highly functional in central nervous system [19]. Large-scale genome-wide (GWAS) association studies conducted in Japanese and Chinese have tried to identify single nucleotide polymorphisms (SNPs) in relation to AIS risk $[19,23]$. In 2015, several new studies which concerned on LBX1 SNPs polymorphisms and AIS susceptibility have been published in Caucasian subjects [24, 25]. The results of these studies showed that several allelic polymorphisms near $L B X 1$ gene may act as potential susceptible factors for AIS, such as rs111090870, rs11598564 and rs625039. However, the results are not all the same and with limited statistical power among these studies. In order to overcome the limitation of single studies, we performed this meta-analysis containing one widely studied locus (rs111090870) and three less studied loci (rs678741, rs11598564 and rs625039), to provide a more comprehensive and precise estimation of $L B X 1$ gene and AIS susceptibility.

\section{Methods}

\section{Data sources}

Six databases were electronically searched, including PubMed, EMBASE, ISI web of knowledge, EBSCO, China National Knowledge Infrastructure (CNKI), and Chinese Biological and Medical Database (CBM), to retrieve studies analyzing the association between AIS susceptibility and $L B X 1$ gene polymorphisms until 1 November 2015. Searching terms were: "adolescent idiopathic scoliosis" or "AIS", in combination with "LBX1" or "ladybird homeobox 1" or "HPX6" or "homeobox", and in combination with: "polymorphism" or "variant" or "genotype" or "allele". We also checked the reference lists of all included studies to make sure no study was missed.

\section{Inclusion criteria}

We first performed initial screening of titles and abstract. A second round screening was based on full-text reviews. Studies were considered eligible if they met the following criteria: (1) It was a case-control study in design; (2) It evaluated the $L B X 1$ gene polymorphisms and AIS susceptibility; (3) AIS was diagnosed on the basis of clinical and radiologic examinations; (4) Individual genotype frequencies or allele frequencies in cases and controls were available.

\section{Exclusion criteria}

Researches were excluded if they met any one of the following criteria: (1) Data came from reviews or abstracts; (2) Genotype and allele frequencies were both unavailable; (3) Repeatedly published literature.

\section{Data extraction and quality assessment}

Two reviewers independently searched and selected literature, and then, extracted relevant data according to a data extraction form. Disagreements were solved by discussion until consensus was made. The extracted data included: the first author, year of publication, country of origin, ethnicity of the study population, genotyping method, source of control, sample size, the genotype and allele frequencies of the $L B X 1$ gene polymorphisms, and information of Hardy-Weinberg equilibrium (HWE) in control group.

Quality assessment was conducted for each article according to a quality evaluation form base on Critical Appraisal Skills Programme (CASP) for case-control study, which containing eleven questions associated with information provided in single studies [26]. Each question has three degrees, "yes" (scored 2), "can't tell" (scored 1 ), or "no" (scored 0). After evaluating each question, a total score from 0 to 22 was given to each article. Studies included in this meta-analysis were divided into 3 grades: Grade A (high quality, scored 15-22), Grade B (medium quality, scored 8-14), Grade C (inferior quality, scored 0-7).

\section{Statistical analysis}

Data analysis was conducted using STATA 11.0 software (Stata Statistical software, College Station, TX, USA, www.stata.com). Odds ratio (OR) and its corresponding $95 \%$ confidence intervals $(95 \% \mathrm{CI})$ were used to evaluate the strength of association between $L B X 1$ gene polymorphisms and AIS susceptibility. Heterogeneity among included studies was tested using chi-square-based Q test and $\mathrm{I}^{2}$ test. $P_{\text {het }}<0.05$ and $I^{2}>50 \%$ were considered as statistically significant for heterogeneity. The MantelHaenszel method was used for fix effect model if no heterogeneity was found. Otherwise, the DerSimonianLaird random effect model was used. Fix effect model considers that across all studies, the genetic factors have similar effects on genetic disorder susceptibility and the observed differences among studies are cause just by chance [27]. Random effect model considers that different studies may have substantial diversity, and it calculates within- as well as between- study difference [28]. Five comparison genetic models were used to assess the association between $L B X 1$ gene polymorphisms and AIS susceptibility. For instance, for rs 1190870 polymorphism, $\mathrm{T}$ allele, we assessed the dominant model $(\mathrm{TT}+\mathrm{CT}$ vs. $\mathrm{CC}$ ), the recessive model (TT vs. TC $+\mathrm{CC}$ ), the allele contrast genetic model ( $\mathrm{T}$ vs. $\mathrm{C}$ ), the heterozygote comparison (CT vs. CC), and the homozygote comparison (TT vs. CC). HWE was tested for included studies if no relevant information was provided in original research. Sensitivity analyses were conducted by omitting individual studies sequentially. Moreover, we performed subgroup analysis 
stratified by ethnicity and gender. Publication bias was quantitatively assessed by Egger's linear regression test [29] and visual inspection of Begg's funnel plots.

\section{Results}

Literature search

We initially identified 86 potentially relevant studies from six databases searched. Firstly, we eliminate duplications, not case-control studies or irrelevant to $L B X 1$ polymorphisms. After this procedure, ten studies were retained. Then, we read the full tests of these articles, and we finally identified 8 case control studies eligible for meta-analysis [1, 19, 23, 24, 30-33], including 10,088 cases and 24,538 controls. One study [34] was excluded for it reported the same datasets as Takahashi et al., but was less detailed. Another study was excluded for genotype and allele frequencies were both unavailable in original research [35]. A flow chart of article selection process is described in Additional file 1: Figure S2.

\section{Studies characteristics}

Table 1 presents the main characteristics of included studies and genotype frequencies of included studies can be found in Additional file 1: Table S1. Of the eight studies, seven [1, 19, 23, 24, 30, 31, 33] were published in English and one [32] was a Chinese doctoral dissertation. There were six studies carried out among Asians [19, 23, 30-33], and two among Caucasians [1, 24]. All studies included were case-control studies in design, and all patients with AIS fulfilled the diagnosis of scoliosis. The number ranged from 94 to 4317 for cases, and 182 to 9823 for controls. Controls were mainly normal healthy populations randomly recruited from general population, who were matched with cases in ethnicity, gender and age. Seven studies containing a total of 13 datasets tested the rs111090870 polymorphism and AIS susceptibility, including seven datasets for female, five for male and one mix gender dataset. Two studies analyzed rs678741, including five datasets for female. Two studies tested rs11598564, and they contained three datasets for female and two for male. Three studies tested rs625039 polymorphism, including three datasets for female, two for male and one mix gender dataset. In quality assessment, all studies included were categorized as grade $\mathrm{A}$, with scores from 15 to 20 (Table 1). Only in one dataset, the genotype distributions in control groups were deviated from HWE.

\section{Quantitative data analysis}

\section{rs111090870 polymorphism and AIS susceptibility}

Seven case-control studies [1, 19, 23, 24, 30-32] containing 13 datasets on relationship between rs111090870 polymorphism and AIS susceptibility were identified, including 5754 cases and 18,628 controls. The results of five genetic models testing rs111090870 polymorphism and AIS susceptibility were showed in Table 2. A significant increase in AIS susceptibility was found in all of five genetic models. In the subgroup analysis stratified by gender, significant increasing AIS susceptibility was found for female in the dominant model (TT + TC vs. CC: OR $=1.13,95 \%$ CI: $1.09-1.16, P<0.001)$ and allele contrast genetic model (T vs. C: $\mathrm{OR}=1.21,95$ \% CI: $1.17-1.25, P<0.001$ ). In mix gender subgroup, allele contrast genetic model also showed a significant increase (T vs. C: $\mathrm{OR}=1.26,95 \%$ CI: 1.09 $1.45, P=0.001)$, but no significant association between rs111090870 and AIS risk was found in male subgroup. In subgroup analyses stratified by ethnicity, significant increasing AIS susceptibility was found for Asians and Caucasians in both genetic models (Table 3). Figure 1 shows the forest plot of allele contrast genetic model testing the association between rs11109070 polymorphism and AIS risk.

\section{rs678741 polymorphism and AIS susceptibility}

Two case-control studies [1,33] containing five datasets on relationship between rs678741 polymorphism and AIS susceptibility were identified, including 4937 cases and 7303 controls, and the five datasets all contained female participants. The results of five genetic models testing rs678741 polymorphism and AIS susceptibility were showed in Table 2. A significant decrease in AIS susceptibility was found in all of five genetic models. In the subgroup analysis stratified by ethnicity, significant decreasing AIS susceptibility was found for both ethnicities in dominant and allele contrast genetic models (Table 3). Figure 2 shows the forest plot of allele contrast genetic model testing the association between rs678741 polymorphism and AIS risk.

\section{rs11598564 polymorphism and AIS susceptibility}

Two case-control studies $[19,30]$ containing five datasets on relationship between rs11598564 polymorphism and AIS susceptibility were identified, including 1966 cases and 13,585 controls and the five databases all contained Asian participants. The results of five genetic models testing rs 11598564 polymorphism and AIS susceptibility were showed in Table 2. A significant increase in AIS susceptibility was found in all of five models except for recessive genetic model (GG vs. GA + AA, OR $=1.09,95 \% \mathrm{CI}: 0.84-1.43, P=0.519$ ). In the subgroup analysis stratified by gender, significant increasing AIS susceptibility was found for both genders in the dominant model and allele contrast genetic model (Table 3). Figure 3 shows the forest plot of allele contrast genetic model testing the association between rs11598564 polymorphism and AIS risk. 
Table 1 Characteristics of the datasets included in meta-analysis on association between LBX1 polymorphisms and AIS

\begin{tabular}{|c|c|c|c|c|c|c|c|c|c|}
\hline First author & Year & Country & Ethnicity & $\begin{array}{l}\text { Case number } \\
\text { (all cases, male/female) }\end{array}$ & $\begin{array}{l}\text { Control number (all } \\
\text { controls, male/female) }\end{array}$ & $\begin{array}{l}\text { Cobb angles degrees } \\
\text { of the included patients }\end{array}$ & Genotyping method & Quality grade & $\begin{array}{l}\text { SNP tested and included } \\
\text { in meta-analysis }\end{array}$ \\
\hline Chettier et al. & 2015 & USA & Caucasian & 620 female cases & 1287 female controls & More than 10 degrees & Affymetrix HuSNP 6.0 Microarray & A (scored 15) & rs111090870, rs678741 \\
\hline Fan et al. & 2012 & China & Asian & $300,52 / 248$ & $788,299 / 489$ & More than 35 degrees & PCR-based invader assay & A (scored 18) & rs111090870 \\
\hline Gao et al. & 2013 & China & Asian & $513,66 / 447$ & $440,151 / 289$ & $25.57 \pm 14.10$ degrees & PCR-MassArray assay & A (scored 16) & $\begin{array}{l}\text { rs111090870, rs11598564, } \\
\text { rs625039 }\end{array}$ \\
\hline Grauers et al. & 2015 & $\begin{array}{l}\text { Sweden and } \\
\text { Denmark }\end{array}$ & Caucasian & $1739,241 / 1498$ & $1812,0 / 1812$ & $38.8 \pm 17.5$ degrees & MassArray assay & A (scored 15) & rs111090870 \\
\hline Jiang et al. & 2013 & China & Asian & $949,129 / 820$ & $976,314 / 662$ & More than 20 degrees & PCR-based invader assay & A (scored 15) & rs111090870 \\
\hline Liu & 2015 & China & Asian & $180,29 / 151$ & $182,30 / 152$ & NA & PCR-MassArray assay & A (scored 17) & rs111090870, rs625039 \\
\hline Takahashi et al. & 2012 & Japan & Asian & $1453,94 / 1359$ & $13127,1849 / 11278$ & More than 15 degrees & $\begin{array}{l}\text { I PCR-based invader assay, } \\
\text { llumina Human610 and Human } \\
\text { Hap550v3 microarrays }\end{array}$ & A (scored 20) & $\begin{array}{l}\text { rs111090870, rs11598564, } \\
\text { rs625039 }\end{array}$ \\
\hline Zhu et al. & 2015 & China & Asian & 4317 female cases & 6016 female controls & $37.2 \pm 9.4$ degrees & $\begin{array}{l}\text { Affymetrix Genome-wide } \\
\text { Human SNP array } 6.0\end{array}$ & A (scored 15) & rs678741 \\
\hline
\end{tabular}

AIS adolescent idiopathic scoliosis; GWAS genome-wide association; USA United States of America; PCR polymorphism chain reaction; SNP single nucleotide polymorphism, NA not applicable 
rs625039 polymorphism and AIS susceptibility

Three case-control studies [19, 30, 32] containing six datasets on relationship between rs678741 polymorphism and AIS susceptibility were identified, including 1 , 646 cases and 13,749 controls, and the six datasets all contained Asians participants. The results of five genetic models testing rs625039 polymorphism and AIS susceptibility were showed in Table 2 . A significant increase in AIS susceptibility was found in all of five genetic models. In the subgroup analysis stratified by gender, significant increasing AIS susceptibility was found for both genders allele contrast genetic models (Table 3). Figure 4 shows the forest plot of allele contrast genetic model testing the association between rs625039 polymorphism and AIS risk.

\section{Sensitivity analysis and publication bias}

Sensitivity analyses were conducted by omitting each dataset sequentially. For four SNP polymorphisms, the result did not change under any genetic model. For rs11090870, when we omitted the male dataset reported by Jiang et al. [31], the indicators for heterogeneity was reduced under dominant, heterozygote, homozygote and allele contrast genetic models. For rs11598564, when we reduce the female dataset reported by Gao et al. [30], the indicators for heterogeneity was reduced under recessive genetic model. Sensitivity analysis suggested that the results for $L B X 1$ gene polymorphisms and AIS susceptibility were stable and statistically robust.

Visual inspection of Begg's funnel plots did not identify substantial asymmetry for all SNPs under any genetic model (Additional file 1: Figure S3-S6). The Egger's linear regression test also indicated no evidence of publication bias in studies testing $L B X 1$ gene polymorphisms and AIS susceptibility $(P>0.05$ for all models tested) (Table 2).

\section{Discussion}

Previous clinical and epidemiological studies found that about $27 \%$ female offspring of female AIS patients would also suffer from AIS, but this phenomenon did not exist in male AIS patients, and that the pairwise concordance rate was higher in monozygotic twin pairs comparing with dizygotic twin pairs [36, 37]. So, the genetic factors play essential roles in pathogenesis of AIS. Preclinical medicine researches shows that, in $L B X 1$ mutant mice, the morphology and neuronal circuitry of the dorsal horn are aberrant and in mice lacking $L B X 1$, cells types that arise in the ventral alar plate acquire more dorsal identities [22, 38]. Therefore, LBX1 is essential for

Table 2 Summary of different genetic model comparison results

\begin{tabular}{|c|c|c|c|c|c|c|c|c|c|}
\hline \multirow[t]{2}{*}{ SNP } & \multirow[t]{2}{*}{ Genetic model } & \multirow[t]{2}{*}{ OR (95 \% Cl) } & \multirow[t]{2}{*}{ Z } & \multirow{2}{*}{$\begin{array}{l}P \\
\text { value }\end{array}$} & \multirow[t]{2}{*}{$P^{2} \%$} & \multirow[t]{2}{*}{$P_{\text {het }}$} & \multirow{2}{*}{$\begin{array}{l}\text { Effect } \\
\text { model }\end{array}$} & \multicolumn{2}{|c|}{ Egger's test } \\
\hline & & & & & & & & $t$ value & $P$ value \\
\hline \multirow[t]{5}{*}{ rs111090870 } & TT+TC vs. CC & $1.12(1.09-1.15)$ & 7.66 & 0.000 & 68.8 & $<0.001$ & $\mathrm{R}$ & 0.42 & 0.684 \\
\hline & $\Pi$ vs. TC $+C C$ & $1.42(1.36-1.49)$ & 15.49 & 0.000 & 42.6 & 0.058 & $\mathrm{~F}$ & 0.92 & 0.378 \\
\hline & TT vs. CC & $1.36(1.27-1.45)$ & 9.07 & 0.000 & 73.2 & $<0.001$ & $\mathrm{R}$ & 0.98 & 0.349 \\
\hline & TC vs. CC & $1.13(1.09-1.18)$ & 5.92 & 0.000 & 56.3 & 0.009 & $\mathrm{R}$ & -0.07 & 0.949 \\
\hline & T vs. C & $1.21(1.16-1.26)$ & 9.52 & 0.000 & 68.7 & $<0.001$ & $\mathrm{R}$ & 0.55 & 0.597 \\
\hline \multirow[t]{5}{*}{ rs678741 } & $\mathrm{GG}+\mathrm{GA}$ vs. $\mathrm{AA}$ & $0.79(0.77-9.82)$ & 16.79 & 0.000 & 0.0 & 0.998 & $\mathrm{~F}$ & 0.85 & 0.458 \\
\hline & GG vs. $G A+A A$ & $0.69(0.64-0.74)$ & 10.98 & 0.000 & 0.0 & 0.880 & $\mathrm{~F}$ & -0.82 & 0.470 \\
\hline & GG vs. AA & $0.68(0.65-0.72)$ & 13.22 & 0.000 & 0.0 & 0.517 & $\mathrm{~F}$ & -1.09 & 0.356 \\
\hline & GA vs. AA & $0.88(0.85-0.91)$ & 8.12 & 0.000 & 0.0 & 0.904 & $\mathrm{~F}$ & -0.73 & 0.519 \\
\hline & G vs. A & $0.83(0.81-0.85)$ & 13.75 & 0.000 & 0.0 & 0.743 & $\mathrm{~F}$ & -0.69 & 0.542 \\
\hline \multirow[t]{5}{*}{ rs11598564 } & $G G+G A$ vs. $A A$ & $1.13(1.10-1.16)$ & 7.98 & 0.000 & 0.0 & 0.649 & $\mathrm{~F}$ & 0.32 & 0.769 \\
\hline & GG vs. $G A+A A$ & $1.09(0.84-1.43)$ & 0.64 & 0.519 & 88.8 & $<0.001$ & $\mathrm{R}$ & 0.29 & 0.726 \\
\hline & GG vs. AA & $1.12(1.33-1.52)$ & 10.12 & 0.000 & 0.0 & 0.761 & $\mathrm{~F}$ & -0.71 & 0.526 \\
\hline & GA vs. AA & $1.13(1.08-1.18)$ & 5.34 & 0.000 & 0.0 & 0.739 & $\mathrm{~F}$ & 1.02 & 0.384 \\
\hline & G vs. A & $1.21(1.16-1.25)$ & 10.03 & 0.000 & 22.2 & 0.273 & $\mathrm{~F}$ & 0.66 & 0.557 \\
\hline \multirow[t]{5}{*}{ rs625039 } & $G G+G A$ vs. $A A$ & $1.07(1.05-1.09)$ & 7.45 & 0.000 & 0.0 & 0.648 & $\mathrm{~F}$ & 0.20 & 0.850 \\
\hline & GG vs. $G A+A A$ & $1.30(1.23-1.37)$ & 9.05 & 0.000 & 0.0 & 0.509 & $\mathrm{~F}$ & 1.18 & 0.303 \\
\hline & GG vs. AA & $1.17(1.13-1.21)$ & 9.48 & 0.000 & 0.0 & 0.651 & $\mathrm{~F}$ & 0.66 & 0.545 \\
\hline & GA vs. AA & $1.09(1.05-1.12)$ & 4.66 & 0.000 & 0.0 & 0.573 & $\mathrm{~F}$ & -0.35 & 0.747 \\
\hline & G vs. A & $1.14(1.11-1.17)$ & 10.14 & 0.000 & 0.0 & 0.584 & $\mathrm{~F}$ & 1.10 & 0.333 \\
\hline
\end{tabular}

SNP single nucleotide polymorphism; $O R$ odds ratio; $C$ confidence interval; $F$ fix-effect model; $R$ random-effect model; $P_{\text {het }} P$ value for heterogeneity $P<0.05$ stands for statistical significance 
Table 3 Results of subgroup analyses

\begin{tabular}{|c|c|c|c|c|c|c|}
\hline \multirow[t]{2}{*}{ SNP } & \multirow[t]{2}{*}{ Comparison } & \multirow{2}{*}{$\begin{array}{l}\text { Number of } \\
\text { datasets }\end{array}$} & \multicolumn{2}{|c|}{ Dominant genetic model } & \multicolumn{2}{|l|}{ Allele contrast } \\
\hline & & & OR $(95 \% \mathrm{Cl})$ & $P$ value & OR $(95 \% \mathrm{Cl})$ & $P$ value \\
\hline \multirow[t]{7}{*}{ rs111090870 } & Gender & & & & & \\
\hline & Female & 7 & $1.13(1.09-1.16)$ & $<0.001$ & $1.21(1.17-1.25)$ & 0.000 \\
\hline & Male & 5 & $1.08(0.93-1.25)$ & 0.319 & $1.15(0.95-1.40)$ & 0.015 \\
\hline & Mix gender & 1 & $1.10(0.99-1.23)$ & 0.087 & $1.26(1.09-1.45)$ & 0.001 \\
\hline & Ethnicity & & & & & \\
\hline & Asian & 10 & $1.13(1.09-1.17)$ & $<0.001$ & $1.22(1.16-1.29)$ & 0.000 \\
\hline & Caucasian & 3 & $1.09(1.08-1.22)$ & $<0.001$ & $1.17(1.14-1.21)$ & 0.000 \\
\hline \multirow[t]{3}{*}{ rs678741 } & Ethnicity & & & & & \\
\hline & Asian & 4 & $0.79(0.77-0.82)$ & $<0.001$ & $0.83(0.81-0.86)$ & 0.000 \\
\hline & Caucasian & 1 & $0.80(0.74-0.86)$ & $<0.001$ & $0.79(0.73-0.86)$ & 0.000 \\
\hline \multirow[t]{3}{*}{ rs11598564 } & Gender & & & & & \\
\hline & Female & 3 & $1.14(1.09-1.16)$ & $<0.001$ & $1.20(1.15-1.25)$ & 0.000 \\
\hline & Male & 2 & $1.16(1.08-1.23)$ & $<0.001$ & $1.27(1.17-1.39)$ & 0.000 \\
\hline \multirow[t]{4}{*}{ rs625039 } & Gender & & & & & \\
\hline & Female & 3 & $1.07(1.05-1.09)$ & $<0.001$ & $1.13(1.10-1.17)$ & 0.000 \\
\hline & Male & 2 & $1.07(1.02-1.13)$ & 0.010 & $1.20(1.12-1.29)$ & 0.000 \\
\hline & Mix gender & 1 & $1.05(0.96-1.14)$ & 0.256 & $1.14(1.02-1.28)$ & 0.025 \\
\hline
\end{tabular}

sensory pathways developments which relay touch and pain. In 1982, Pincott et al. found that the loss of proprioceptive innervation could result in asymmetrical weakness of the paraspinal muscles, and which may finally cause scoliosis [39]. As a result, it is quite reasonable to deduce that $L B X 1$ gene may be a target spot in pathogenesis of AIS.

Case-control study is a useful tool to detect gene and disease susceptibility. GWAS is a kind of case-control cohort study in essential, which is a powerful method to

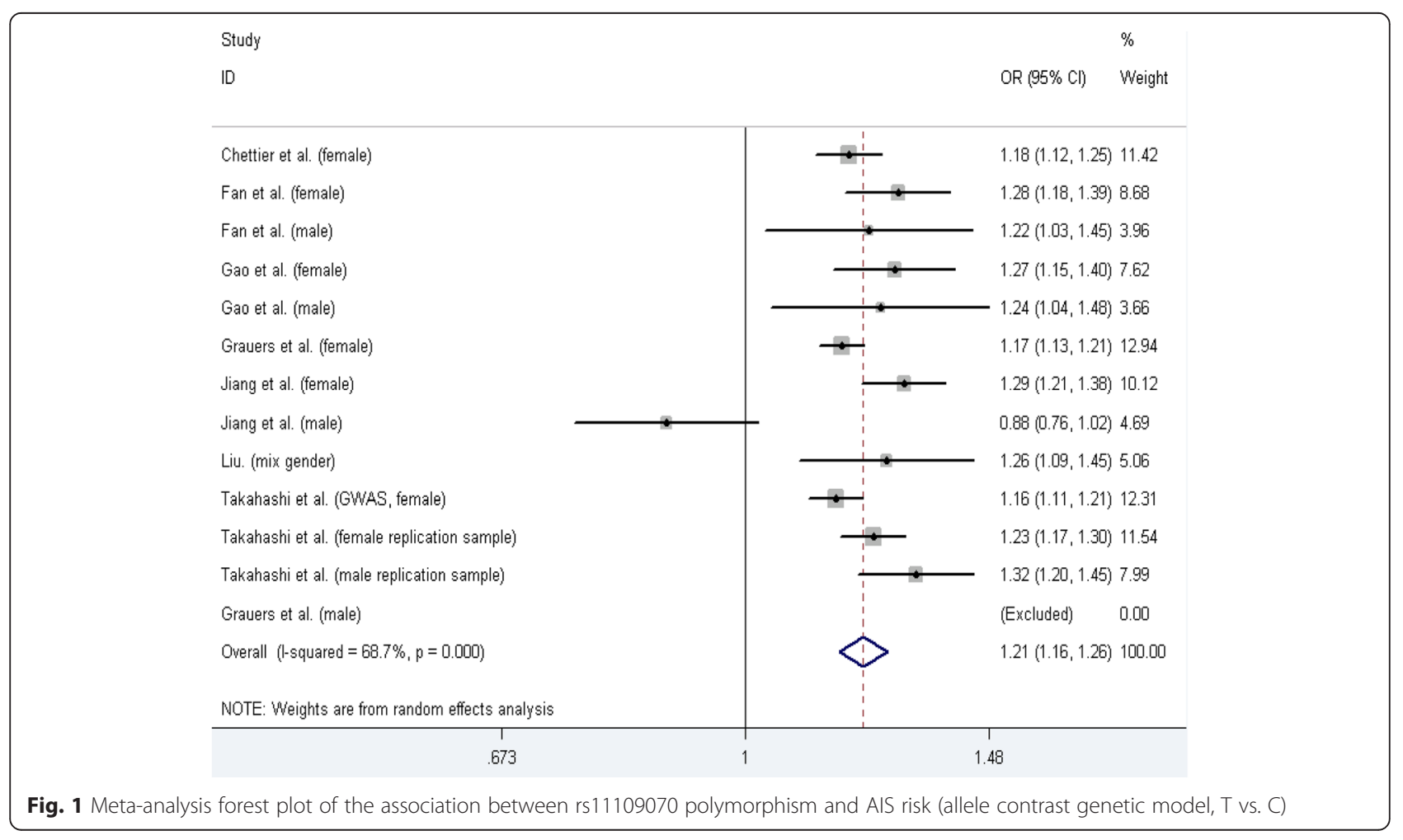




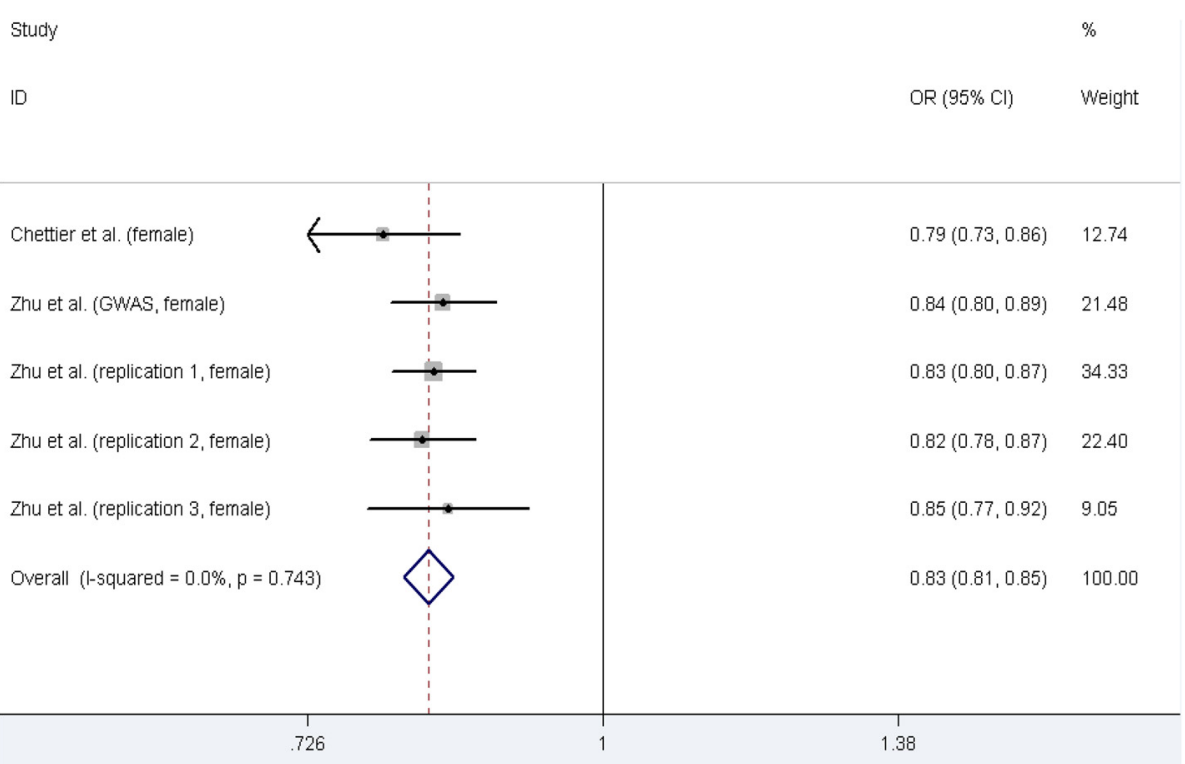

Fig. 2 Meta-analysis forest plot of the association between rs678741 polymorphism and AlS risk (allele contrast genetic model, G vs. A)

identify genetic association with diseases and has been applied in genetic predisposition studies increasingly. Therefore, GWAS is widely used in AIS susceptibility study, to identity risk genes for this most common and complex musculoskeletal system polygenic disease [35, 40, 41].
Fan et al. and Takahashi et al. [19, 23] first reported results of a GWAS analyzing the association between LBX1 gene and AIS susceptibility in 2012 and their results showed statistical significant associations for rs111090870, rs11598564 and rs625039. From 2013 to

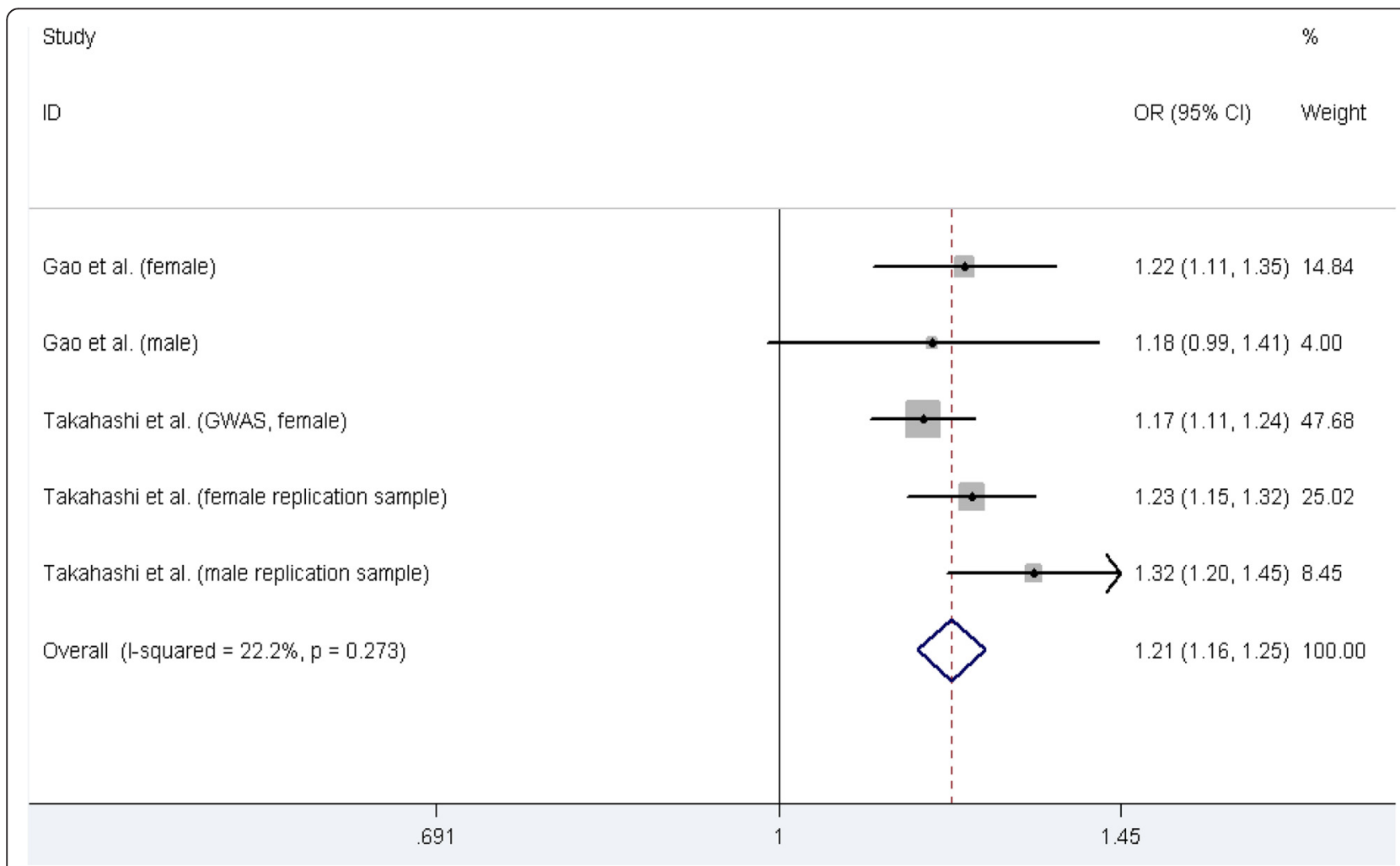

Fig. 3 Meta-analysis forest plot of the association between rs11598546 polymorphism and AIS risk (allele contrast genetic model, G vs. A) 


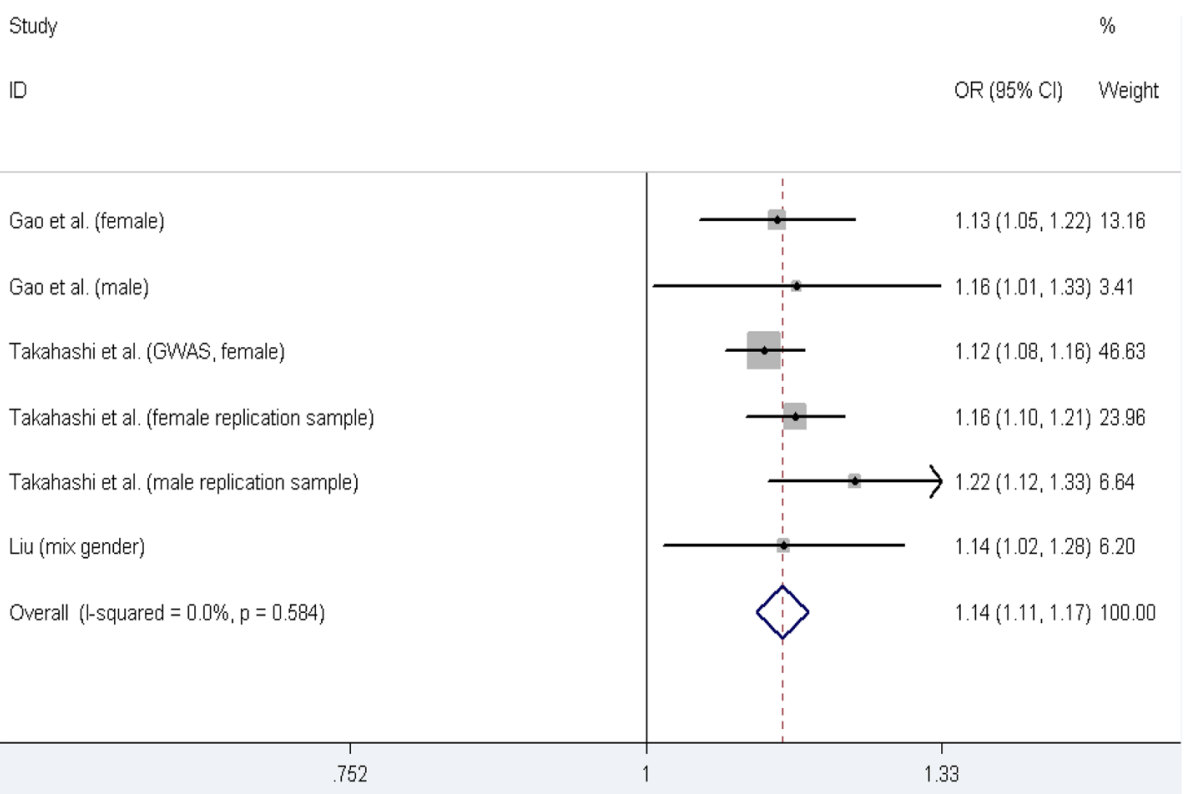

Fig. 4 Meta-analysis forest plot of the association between rs625039 polymorphism and AlS risk (allele contrast genetic model, G vs. A)

2015, several subsequent studies have conducted to investigate the $L B X 1$ polymorphisms in pathogenesis of AIS. However, the results are inconsistent, with some datasets found positive associations, and other find no relevance or even negative association. Several reasons, including different recruitment criteria, subjects' characteristics, sample size, different ethnic population and gender, may lead to the inconsistency.

In order to avoid limitations of individual case-control or GWA study, we conducted this meta-analysis to pool the findings of rs111090870, rs678741 rs11598564 and rs625039 polymorphisms in all original studies. As we reported in result part, the eight included studies were of high quality judged by CASP standard. They clearly reported their participants choosing criteria, sample sizes, characteristics of cases and controls and genotyping methods. Moreover, except one dataset cannot be tested for HWE, only one dataset was deviated from HWE in the control group. Gender and ethnicity were matched in case and control group. The results showed that $\mathrm{T}$ allele of rs111090870 is significant associated with increased AIS susceptibility in Asians ( $\mathrm{T}$ vs. C, $\mathrm{OR}=1.22$, $95 \% \mathrm{CI}: 1.16-1.29, P<0.001)$, Caucasians (T vs. $\mathrm{C}, \mathrm{OR}=1.17,95 \% \mathrm{CI}: 1.14-1.21, P<0.001$ ) and in female (T vs. $\mathrm{C}, \mathrm{OR}=1.21,95 \% \mathrm{CI}: 1.17-1.25, P<0.001$ ). The $\mathrm{G}$ allele of rs678741 decreased AIS risk in female (G vs. A, OR $=0.83,95 \%$ CI: $0.81-0.85, P<0.001$ ), and the G allele of the rs625039 polymorphism may increase AIS susceptibility in Asians (G vs. A, OR $=1.14,95 \% \mathrm{CI}$ : $1.11-1.17, P<0.001)$. For the rs11598564 polymorphism, $G$ allele may also increase AIS risk, but in recessive model, no statistical significant association was detected and the result was with heterogeneity. So, the result of rs11598564 should be interpreted with caution.

We should notice the heterogeneity existed in this meta-analysis. For the rs111598564 and rs625039 polymorphisms, no heterogeneity was found among studies. However, in the rs111090870 polymorphism, significant heterogeneity was found in all models except the recessive model, and for the rs11598564 polymorphism, heterogeneity was detected in recessive genetic models. For rs111090870 polymorphism, the heterogeneity detected in four genetic models was effectively decreased in sensitivity analysis when the male dataset in study by Jiang et al. [31] was omitted. In this study, the genotype distribution in control group was deviated from HWE. For rs11598564 polymorphism, when we stratified by gender, the heterogeneity existed in female group only, and when omitted individual dataset sequentially, we find female group in study by Gao et al. [30] contributed to the heterogeneity. Moreover, the removal of these datasets did not materially change the overall results of any genetic models. Therefore, deviation from HWE and different genders may contribute to overall heterogeneity of this meta-analysis.

Two previous meta-analyses [42, 43] have tried to analyze the association between rs111090870 polymorphism and AIS susceptibility in East Asians. For these two studies, all of the genetic models provided statistically significant comparison results. For instance, the ORs and their corresponding $95 \% \mathrm{CI}$ of the dominant models were $2.04(2.27-3.03)$ and $2.02(1.78-2.30)$ respectively. The results of our study were quite similar to theses to studies, but the effect sizes were lower than theirs, as the effect 
size of the dominant model in our study were 1.13 (1.091.17) for Asians and 1.12 (1.09-1.15) for overall population. Comparing with them, our meta-analysis has some important improvements. For rs111090870 polymorphism, some new published researches were included in our meta-analysis polymorphism, and through strict methodological process, we provided a more comprehensive view of included studies. The above mentioned meta-analysis only focus on east Asian participants, but our study also included Caucasian subjects and in subgroup studies, we stratified by ethnicity to test if there existed differences in variant ethnicities. For rs678741, rs11598564 and rs625039 polymorphisms, to the best of our knowledge, no published combined study has detected their association with AIS.

Several limitations of this study may affect the results. Firstly, only published English and Chinese studies were included in this meta-analysis and we only included published studies from six databases. Relevant studies in other languages and databases may have been missed. Secondly, in our meta-analysis, all datasets included in rs11598564 and rs625039 were based on Asian subjects. Additional researches in other ethnicities are needed to generalize our finding for rs 11598564 and rs625039. For rs678741, all included datasets were based on female subjects, and other researches for males are needed. Because the same polymorphism may act differently in different ethnical backgrounds genders, results from rs678741 cannot extend to male and results from rs11598564, rs625039 cannot extend to Caucasians or other ethnicities. Thirdly, most included studies did not distinguish between the magnitude or phenotype of scoliosis and genotyping results. Therefore, we were unable to provide different pooled ORs according to different magnitude or phenotype of AIS. Finally, the possible ethiology pathogenesis of AIS is complex, but due to insufficiency of included studies, we did not detect the interactions between genetic factors and other factors. Considering that meta analysis is a kind of retrospective research and may easily be affected by methodological deficiencies of included studies, we developed a detailed protocol before conducting this analysis, to ensure the quality of our meta-analysis.

\section{Conclusion}

From the combination results of currently included studies, our meta-analysis suggested that the $\mathrm{T}$ allele of rs111090870 polymorphism near $L B X 1$ gene is significant associated with increased AIS susceptibility in Asians, Caucasians and in female. The G allele of rs678741 may decrease AIS risk in female and the G allele of rs625039 polymorphism may increase AIS susceptibility in Asians. More studies with multiple ethnics and different genders are needed to generalize the results.

\section{Additional file}

Additional file 1: Table S1. Genotype and allele distribution of LBX1 polymorphisms in AIS cases and controls. Figure S1. X-ray of an AIS patient. Figure S2. Flow chart of data selection. Figure S3. Begg's funnel plot for publication bias in the dominant model for rs111090870 polymorphism. Figure S4. Begg's funnel plot for publication bias in the dominant model for rs678741 polymorphism. Figure S5. Begg's funnel plot for publication bias in the dominant model for rs11598564 polymorphism. Figure S6. Begg's funnel plot for publication bias in the dominant model for rs625039 polymorphism. (DOCX 144 kb)

\section{Abbreviations}

AA-NAT, arylalkylamine N-acetyltransferase; AIS, adolescent idiopathic scoliosis; BNC2, Basonuclin 2; CASP, Critical Appraisal Skills Programme; CBM, Chinese Biological and Medical Database; Cl, confidence interval; CNKI, China National Knowledge Infrastructure; GWAS, Large-scale genome-wide; HWE, HardyWeinberg equilibrium; LBX1, ladybird homeobox 1; OR, odds ratios; PCR, Polymorphism chain reaction; SNP, single nucleotide polymorphism; TPH1, tryptophan hydroxylase 1

\section{Acknowledgments}

We would like to acknowledge all authors of the original studies included in this meta-analysis.

\section{Funding}

This work was not supported by any fund.

\section{Availability of data and materials}

The datasets supporting the conclusions of this article are included within the article and in the Additional file 1.

\section{Authors' contributions}

YC was the first author who conceived the study, took part in protocol making, articles searching, data extraction, data analyzing, and wrote the first draft of the manuscript. JM was the second author and corresponding author, who contributed to the article selection, data extraction, final interpretation, and commented on successive versions of the manuscript. QZ took part in protocol revising, article selection and helped in manuscript writing. $\mathrm{HL}$ and $\mathrm{HL}$ together produced the figures and tables in article, arbitrated studies for inclusion and commented on successive drafts of the manuscript. All authors read and approved the final manuscript.

\section{Competing interests}

The authors declare that they have no competing interests. The authors alone are responsible for the content and the writing of the paper.

\section{Consent for publication}

Written informed consent was obtained from the parents of patient for publication of the accompanying image in Additional file 1.

Ethics approval and consent to participate

Not applicable as this is a meta-analysis of previously published papers.

Received: 3 January 2016 Accepted: 29 June 2016

Published online: 22 July 2016

\section{References}

1. Chettier R, Nelson L, Ogilvie JW, Albertsen HM, Ward K. Haplotypes at LBX1 have distinct inheritance patterns with opposite effects in adolescent idiopathic scoliosis. PLoS One. 2015;10(2):e0117708.

2. Weinstein SL, Dolan LA, Cheng JC, Danielsson A, Morcuende JA. Adolescent idiopathic scoliosis. Lancet. 2008;371(9623):1527-37.

3. Stokes IA. Three-dimensional terminology of spinal deformity. A report presented to the Scoliosis Research Society by the Scoliosis Research Society Working Group on 3-D terminology of spinal deformity. Spine. 1994;19(2):236-48.

4. Perdriolle R, Le Borgne P, Dansereau J, de Guise J, Labelle H. Idiopathic scoliosis in three dimensions: a succession of two-dimensional deformities? Spine. 2001;26(24):2719-26. 
5. Dreimann M, Hoffmann M, Kossow K, Hitzl W, Meier O, Koller H. Scoliosis and chest cage deformity measures predicting impairments in pulmonary function: a cross-sectional study of 492 patients with scoliosis to improve the early identification of patients at risk. Spine. 2014;39(24):2024-33.

6. Newton PO, Faro FD, Gollogly S, Betz RR, Lenke LG, Lowe TG. Results of preoperative pulmonary function testing of adolescents with idiopathic scoliosis. A study of six hundred and thirty-one patients. J Bone Joint Surg Am. 2005;87(9):1937-46.

7. White SF, Asher MA, Lai SM, Burton DC. Patients' perceptions of overall function, pain, and appearance after primary posterior instrumentation and fusion for idiopathic scoliosis. Spine. 1999;24(16):1693-9. discussion 1699-1700.

8. Misterska E, Glowacki M. Assessment of pain severity and function of lumbar spine in idiopathic scoliosis. Ortopedia Traumatologia Rehabilitacja. 2009; 11(5):433-7.

9. McMaster ME, Lee AJ, Burwell RG. Physical activities of Patients with adolescent idiopathic scoliosis (AIS): preliminary longitudinal casecontrol study historical evaluation of possible risk factors. Scoliosis. 2015;10:6.

10. Kulis A, Gozdzialska A, Drag J, Jaskiewicz J, Knapik-Czajka M, Lipik E, Zarzycki D. Participation of sex hormones in multifactorial pathogenesis of adolescent idiopathic scoliosis. Int Orthop. 2015;39(6):1227-36.

11. Li W, Li Y, Zhang L, Guo H, Tian D, Li Y, Peng Y, Zheng Y, Dai Y, Xia K, et al. AKAP2 identified as a novel gene mutated in a Chinese family with adolescent idiopathic scoliosis. J Med Genet. 2016;53(7):448-93.

12. Xu JF, Yang GH, Pan XH, Zhang SJ, Zhao C, Qiu BS, Gu HF, Hong JF, Cao L, Chen $Y$, et al. Association of GPR126 gene polymorphism with adolescent idiopathic scoliosis in Chinese populations. Genomics. 2015;105(2):101-7.

13. Zhang Y, Gu Z, Qiu G. The association study of calmodulin 1 gene polymorphisms with susceptibility to adolescent idiopathic scoliosis. BioMed Res Int. 2014;2014:168106

14. Bae JW, Cho CH, Min WK, Kim UK. Associations between matrilin-1 gene polymorphisms and adolescent idiopathic scoliosis curve patterns in a Korean population. Mol Biol Rep. 2012;39(5):5561-7.

15. Zhao D, Qiu GX, Wang YP, Zhang JG, Shen JX, Wu ZH. Association between adolescent idiopathic scoliosis with double curve and polymorphisms of calmodulin1 gene/estrogen receptor-alpha gene. Orthop Surg. 2009;1(3): 222-30.

16. Ogura Y, Kou I, Miura S, Takahashi A, Xu L, Takeda K, Takahashi Y, Kono K, Kawakami N, Uno K, et al. A functional SNP in BNC2 is associated with adolescent idiopathic scoliosis. Am J Hum Genet. 2015;97(2):337-42.

17. Yang $P$, Liu $H$, Lin J, Yang $H$. The association of rs4753426 polymorphism in the melatonin receptor 1B (MTNR1B) gene and susceptibility to adolescent idiopathic scoliosis: a systematic review and meta-analysis. Pain Phys. 2015; 18(5):419-31.

18. Wang H, Wu Z, Zhuang Q, Fei Q, Zhang J, Liu Y, Wang Y, Ding Y, Qiu G. Association study of tryptophan hydroxylase 1 and arylalkylamine Nacetyltransferase polymorphisms with adolescent idiopathic scoliosis in Han Chinese. Spine. 2008;33(20):2199-203.

19. Takahashi Y, Kou I, Takahashi A, Johnson TA, Kono K, Kawakami N, Uno K, Ito $\mathrm{M}$, Minami $\mathrm{S}$, Yanagida $\mathrm{H}$, et al. A genome-wide association study identifies common variants near LBX1 associated with adolescent idiopathic scoliosis. Nat Genet. 2011;43(12):1237-40.

20. Xu J, Nonogaki M, Madhira R, Ma HY, Hermanson O, Kioussi C, Gross MK. Population-specific regulation of Chmp2b by Lbx1 during onset of synaptogenesis in lateral association interneurons. PLoS One. 2012;7(12): e48573.

21. Jagla K, Dolle P, Mattei MG, Jagla T, Schuhbaur B, Dretzen G, Bellard F, Bellard M. Mouse Lbx1 and human LBX1 define a novel mammalian homeobox gene family related to the Drosophila lady bird genes. Mech Dev. 1995;53(3):345-56.

22. Gross MK, Dottori M, Goulding M. Lbx1 specifies somatosensory association interneurons in the dorsal spinal cord. Neuron. 2002;34(4):535-49.

23. Fan $Y H$, Song $Y Q$, Chan D, Takahashi $Y$, Ikegawa S, Matsumoto M, Kou I, Cheah KS, Sham P, Cheung KM, et al. SNP rs11190870 near LBX1 is associated with adolescent idiopathic scoliosis in southern Chinese. J Hum Genet. 2012;57(4):244-6.

24. Grauers A, Wang J, Einarsdottir E, Simony A, Danielsson A, Akesson K, Ohlin A, Halldin K, Grabowski P, Tenne M, et al. Candidate gene analysis and exome sequencing confirm LBX1 as a susceptibility gene for idiopathic scoliosis. Spine J. 2015;15(10):2239-46.
25. Wise CA, Gao X, Shoemaker S, Gordon D, Herring JA. Understanding genetic factors in idiopathic scoliosis, a complex disease of childhood. Curr Genomics. 2008;9(1):51-9.

26. Critical Appraisal Skills Programme (CASP) 2014. 11 questions to help you make sense of Case Control Study. [http://media.wix.com/ugd/dded87_ 63fb65dd4e0548e2bfd0a982295f839e.pdf]. Accessed 1 Nov 2015.

27. DerSimonian R, Laird N. Meta-analysis in clinical trials. Control Clin Trials. 1986;7(3):177-88.

28. Higgins JP, Thompson SG. Quantifying heterogeneity in a meta-analysis. Stat Med. 2002;21(11):1539-58.

29. Egger M, Davey Smith G, Schneider M, Minder C. Bias in meta-analysis detected by a simple, graphical test. BMJ. 1997;315(7109):629-34.

30. Gao W, Peng Y, Liang G, Liang A, Ye W, Zhang L, Sharma S, Su P, Huang D. Association between common variants near LBX1 and adolescent idiopathic scoliosis replicated in the Chinese Han population. PLoS One. 2013;8(1):e53234

31. Jiang H, Qiu X, Dai J, Yan H, Zhu Z, Qian B, Qiu Y. Association of rs 11190870 near LBX1 with adolescent idiopathic scoliosis susceptibility in a Han Chinese population. Eur Spine J. 2013;22(2):282-6.

32. Liu S. Association study of gene polymorphisms with adolescent idopathic scoliosis susceptibility in a Northern Han Chinese population. (Doctor Thesis). Doctor. China: Peking Union Medical College; 2015.

33. Zhu Z, Tang NL, Xu L, Qin X, Mao S, Song Y, Liu L, Li F, Liu P, Yi L, et al. Genome-wide association study identifies new susceptibility loci for adolescent idiopathic scoliosis in Chinese girls. Nat Commun. 2015;6:8355.

34. Miyake A, Kou I, Takahashi Y, Johnson TA, Ogura Y, Dai J, Qiu X, Takahashi A, Jiang $\mathrm{H}$, Yan $\mathrm{H}$, et al. Identification of a susceptibility locus for severe adolescent idiopathic scoliosis on chromosome 17q24.3. PLoS One. 2013; 8(9):e72802.

35. Sharma S, Gao X, Londono D, Devroy SE, Mauldin KN, Frankel JT, Brandon JM, Zhang D, Li QZ, Dobbs MB, et al. Genome-wide association studies of adolescent idiopathic scoliosis suggest candidate susceptibility genes. Hum Mol Genet. 2011;20(7):1456-66.

36. Andersen $\mathrm{MO}$, Thomsen $\mathrm{K}$, Kyvik $\mathrm{KO}$. Adolescent idiopathic scoliosis in twins: a population-based survey. Spine. 2007;32(8):927-30.

37. Harrington PR. The etiology of idiopathic scoliosis. Clin Orthop Relat Res. 1977;126:17-25.

38. Muller T, Brohmann $H$, Pierani A, Heppenstall PA, Lewin GR, Jessell TM, Birchmeier C. The homeodomain factor Ibxl distinguishes two major programs of neuronal differentiation in the dorsal spinal cord. Neuron. 2002;34(4):551-62.

39. Pincott JR, Taffs LF. Experimental scoliosis in primates: a neurological cause. J Bone Joint Surg (Br). 1982;64(4):503-7.

40. Kingsmore SF, Lindquist IE, Mudge J, Beavis WD. Genome-wide association studies: progress in identifying genetic biomarkers in common, complex diseases. Biomark Insights. 2007;2:283-92.

41. McCarthy Ml, Abecasis GR, Cardon LR, Goldstein DB, Little J, loannidis JP, Hirschhorn JN. Genome-wide association studies for complex traits: consensus, uncertainty and challenges. Nat Rev Genet. 2008;9(5):356-69.

42. Chen S, Zhao L, Roffey DM, Phan P, Wai EK. Association of rs 11190870 near LBX1 with adolescent idiopathic scoliosis in East Asians: a systematic review and meta-analysis. Spine J. 2014;14(12):2968-75.

43. Liang J, Xing D, Li Z, Chua S, Li S. Association between rs11190870 polymorphism near LBX1 and susceptibility to adolescent idiopathic scoliosis in East Asian population: a genetic meta-analysis. Spine. 2014. [Epub ahead of print].

\section{Submit your next manuscript to BioMed Central and we will help you at every step:}

- We accept pre-submission inquiries

- Our selector tool helps you to find the most relevant journal

- We provide round the clock customer support

- Convenient online submission

- Thorough peer review

- Inclusion in PubMed and all major indexing services

- Maximum visibility for your research

Submit your manuscript at www.biomedcentral.com/submit 